\title{
Study of Optimization and Design for Disk-Type Molecular Pump Based on DSMC Method
}

\author{
Zhou Bo, Guo Zixue, Xie Quanxin, and Ran Bo
}

\begin{abstract}
The DSMC method was applied to accomplish the simulative calculation of the performance of the disk-type molecular pump for $\mathrm{N} 2$ and the effect of the main mechanical parameters of its channels while the parameters of the external structure were fixed. The main problems in using DSMC method such as the setting of the boundary conditions, which especially cater to the high rotative speed condition was solved. A new subassembly with optimized mechanical parameters was give out based on the calculation result.
\end{abstract}

Index Terms-Disk-type molecular pump, DSMC method, simulative, $\mathbf{N 2}$.

\section{INTRODUCTION}

A common disk-type molecular pump mainly consisted of two parts-the static disk and the dynamic disk. Several channels were settled on the static disk to force the gas moving. The disk-type molecular pump for calculating was an assembly of a complicated machine, so its external structure was fixed in order to facilitate the assembly work. According to its dimension and the actual pressure of $N_{2}$, which was the main only active gas, in its working area, the gaseous flow field was in the transition flow region. The DSMC method [1]-[7] was applicable for this kind of problem and had a much more accurate result, so it was used.

Additionally, the channels of the pump were circle curve in shape with rectangular sections. All the channels are circular symmetrical, so in the following sections it was mainly discussed about the flow field of a single channel on the static disk. Furthermore, the pressure at the outlet and the gas leakage rate at the inlet were both kept stable while calculating, in other words, they were constants in the calculation.

The performance of the molecular pump was measured by the pressure ratio which meant the ratio of the outlet's pressure to the inlet's.

(A domestic patent in China PR is going to be applied for this molecular pump in the near future; as a result all the mechanical parameters could not be mentioned in this paper according to the related laws. Unfortunately, all of them have to be obscure or modified in this paper.)

Manuscript received March 1, 2013; revised May 17, 2013.

Zhou Bo is with the Department of Engineering Physics, Tsinghua Univercity, Beijing, China (e-mail: bozhou.ipce@gmail.com).

Guo Zixue and Ran Bo are with the Institute of Physical and Chemical Engineering, Tianjin, China (e-mail: guozx605@139.com, danb03@163.com).

Xie Quanxin is with the Physical Department of Moscow Engineering Physics Institute, State University, Moscow, Russian (e-mail: xxm74@163.com).

\section{Simulative CAlculation Process}

\section{A. Modeling of the Channel}

A single channel on the static disk is schematically presented in Fig. 1. $R_{1}$ and $R_{2}$ mean the foregoing fixed radius of the disk. As it was described in the introductions, the channel is circle curve in shape, and the $R c$ meant the radius of the circle. All the centers of the circles are also on the same circle, its radius is denoted by $R_{3}$. The central angle of the adjoining circles is denoted by $\theta$.

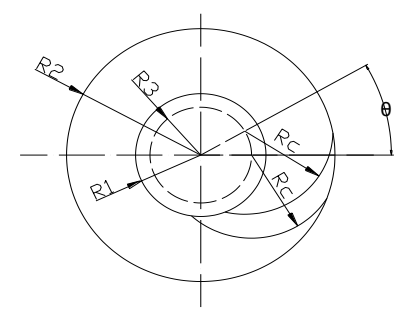

Fig. 1. Plan of a single channel on the static disk.

Obviously, $\theta, R c$ and $R_{3}$ are the most important parameters to optimize, because they define the shape of the channel when the external structure of the disk were fixed. But $\theta$ were determined by the number of the channel and the machining technic, and in most conditions its varying range was not too large. In this calculation $\theta$ could also be evaluated by a common quantity.

There is another key parameter unshown in the plan, it is the depth of the channel. In the calculation it is denoted by $h$. especially the depth is the answer of adding the real depth of the channel and the gap between the static disk and the dynamic disk.

Separating $R c, R_{3}$ and h averagely to mesh the model of the channel into the proper form [8] for the DSMC method as the Fig. 2 presents.

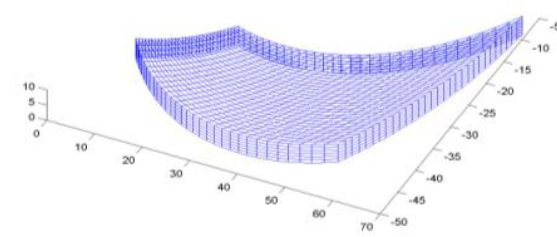

Fig. 2. Sketch of the meshing on the geometric model of a single channel.

\section{B. Calculation Settings}

As the working condition was only one kind of gas (N2) flowing at the normal temperature, the VHS [1], [3], [9] model was applied because of its suitability and economy. At 
the same time, the NTC [9] method advanced by Bird was utilized to sample the collision.

The Mersenne Twister method [10], [11] advanced by Makoto Matsumoto and Takuji Nishimura was applied to generate the random number used in the calculation.

As mentioned in the former sections, the depth of the channel model consisted of two parts-the real depth of the channel and the gap between the static disk and the dynamic disk. In the calculation while the virtual molecule entered the gap and collided with its walls, it meant the virtual molecule would have left the channel. As all the virtual molecules had gotten a very high rotative initial speed which was about more than $300 \mathrm{~m} / \mathrm{s}$ from the rotating dynamic disk, the thickness of the wall between the two adjoining channels could be ignored because it was too small comparing with the very high speed. As a result when one virtual molecule moved out, it could be assumed that another virtual molecule with the similar status which only rotated by an angel of $\theta$ from the exiting one entered the channel from the other wall. In simple words, when a molecule left the channel into its adjoining channel, another equivalent molecule must left the other adjoining channel into it due to the ignoring of the thickness between them.

After applying the assumption of the gap, the calculation could be more complicated. A time value for judging the movement and position of the molecule was necessary. It was denoted by $t_{j d g}$ which was the minimum time among the times of the molecule's moving to the walls of the channel and its remaining moving time in a single time step. According to the value of $t_{j d g}$, the judgement of the molecule's position and movement could be simplified.

\section{NUMERICAL RESULTS}

\section{A. Effects of Single Parameter}

In the calculation, first only change one parameter while fix the other two in order to discuss the effects of it on the performance of the pump. The numerical results were shown in Fig. 3-Fig. 5.

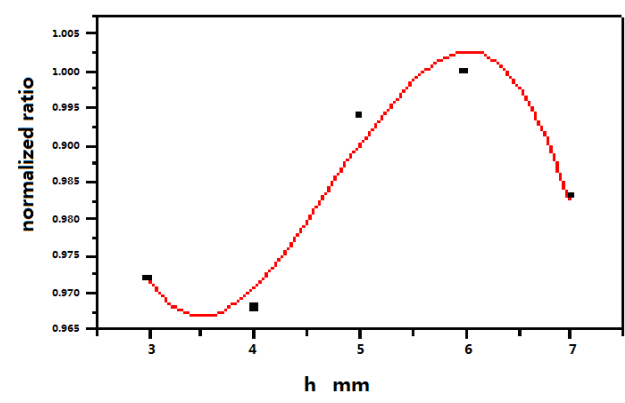

Fig. 3. Varying of the pressure ratio with $\mathrm{h}$ increasing.

The depth of the channel denoted by $h$ has a great effect on the perpendicular aspect of the channel, and its value means whether channel is too deep or too shallow. Similarly, $R_{3}$ has a great effect on the shape of the channel, and its value means whether the channel is too straight or too bended. $R c$ also defines the shape of the channel, but its effect is much more less than $R_{3}$ 's. So the pressure ratio varies more obviously as $\mathrm{h}$ and $R_{3}$ vary, but less obviously as $R c$ varies.

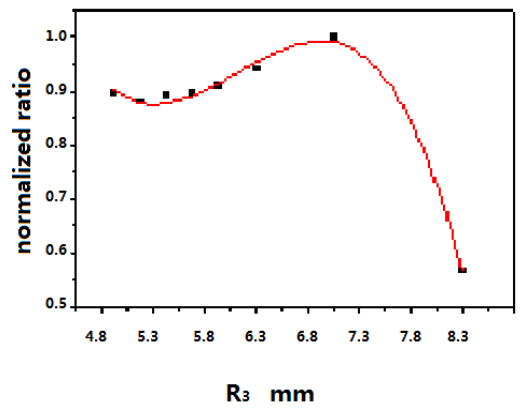

Fig. 4. Varying of the pressure ratio with $R_{3}$ increasing.

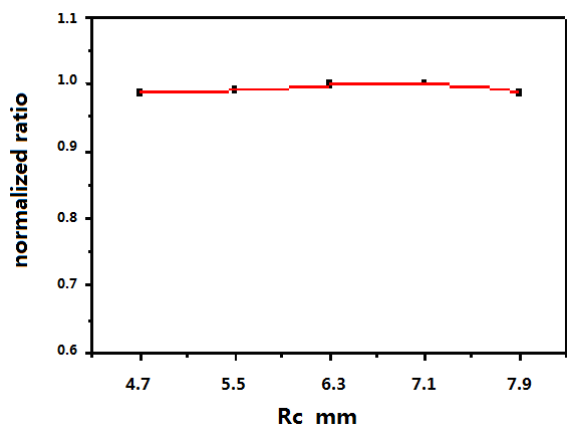

Fig. 5. Varying of the pressure ratio with $R c$ increasing.

When the depth is too shallow, the collisions between the channel and the molecules are too rare, but when it is too deep, too many molecules will stay in the channel. In both conditions, the channel's guiding effect is weakened. By the same meaning, when the channel is too straight or too bended, its guiding effect is weakened too. So $h$ and $R_{3}$ have an optimized value each as the figures show.

\section{B. Effects of Combined Parameters}

$R c$ and $R_{3}$ define the shape of the channel together, and $h$ defines the depth. So the optimization of $h$ could be confirmed first according its effect on the pressure ratio. But the optimization of $R c$ and $R_{3}$ has to be calculated in the conditions that both of them are varying. The numerical result is shown as Fig. 6.

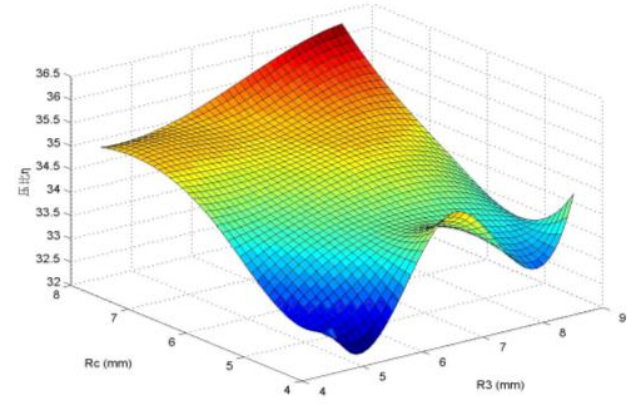

Fig. 6. Combined influence of $R_{3}$ and $R c$ on the pressure ratio.

Obviously, there is an optimized combination of the $R c$ and $R_{3}$, and combining this result with the optimization of $h$, a group of optimized parameters was confirmed.

\section{CONCLUSION}

The effects of the key structural parameters of the disk-type molecular pump such as the depth of the channel denoted by $h$, the radius of the channel's circle denoted by $R c$ 
and the radius of the circle which the center of the channel's circle laid on denoted by $R_{3}$ was calculated via DSMC method.

As a conclusion, $h$ and $R_{3}$ has a great effect on the performance of the molecular pump but $R c$ has a little one. While $h$ or $R_{3}$ increasing and other parameters are fixed, there both appear an obvious extremum of the pressure ratio of the molecular pump. Additionally, the combination of $R_{3}$ and $R c$ also has an optimization.

According to the calculation, there is an optimization of the combination of $R c, R_{3}$ and $h$. In this combination, $h$ is valued as the extremum while it was optimized exclusively; $R c$ and $R_{3}$ were valued as their optimized combination.

\section{REFERENCES}

[1] Q. Shen, "Rarefied Gas dynamics," Industry of National Defence, Jan. 2003.

[2] G. A. Bird, "Recent advances and current challenges for DSMC," Computers Math. Applic., vol. 35, no. 1/2, pp.1-14 ,1998.

[3] G. A. Bird, Molecular gas dynamics and the direct simulation of gas flows, Oxford University Press, 1994.

[4] M. S. Woronowicz and D. F. G. Rault, "Cecignani-Lampis-Lord gas-surface interaction model: Comparitions between theory and simulation," J. Spacecraft and Rockets, vol. 31, no. 532, 1994.

[5] W. Wagner, "A convergence proof for Bird's direct simulation Monte Carlo method for the Boltzmann equation," J. Star. Phys., vol. 66, pp. $1011,1992$.

[6] M. Pulvirenti, W. Wagner, and M. B. Zavelani, "Convergence of particle schemes for the Boltzmann equation," Euro. J. Mech., vol. B7, pp. 339, 1990.

[7] H. A. Babovsky, "Convergence proof for Nanbu's Boltzmann simulation scheme," Euro. J. Mech. B: Fluids, vol. 8, no. 1, pp. 41-45, 1989.

[8] G. A. Bird, Molecular Gas Dynamics, Oxford: Clarenton Press, 1976.

[9] G. A. Bird, "Monte Carlo simulation in an engineering context," Progr. Astro. Aero., in Proc. International Symposium on Rarefied Gas Dynamics, vol. 74, 1981, pp. 239-255.
[10] M. Matsumoto and T. Nishimura, "Mersenne Twister: A 623-dimensinally equi-distributed Uniform Pseudo-Random Number Generater," ACM Transactions on Modeling and Computer Simulation, vol. 8, no. 1, January 1998.

[11] M. Matsumoto and T. Nishimura, "Sum-Discrepancy test on pseudorandom number generators," Mathematics and Computers in Simulation, vol. 62, no. 2003, pp. 431-442, 2003.

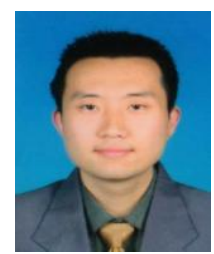

Zhou Bo was born in Changchun, Jilin Province, China PR, on Dec. 27, 1981. He got his bachelor degree from Department of Engineering Physics, Tsinghua Univercity, Beijing, China PR, 2005. His major field of study is gas dynamics.

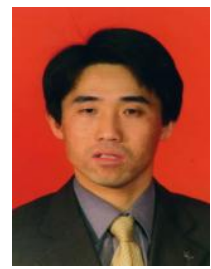

Guo Zixue was born in Bo-Xing, Shandong Provinc, China PR on Dec. 27, 1972. He got his master dgree from Institute of Physical and Chemical Engineering ,Tianjin, China PR, 1998. His maijor field of study is gas dynamics.

Xie Quanxin was born in Hunan province, China PR, on Agu. 26, 1974. He got his Phd degree of maths and physics from physical department of Moscow Engineering Physics Institute, State University, Moscow, Russian, 2007. His major field of study is molecular physics.

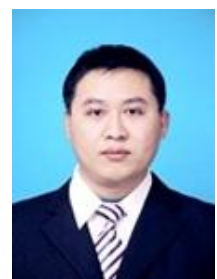

Ran Bo was born in Shehong, Sichuan Province, China PR, on Jan. 4, 1984. He got his master degree from Institute of Physical and Chemical Engineering ,Tianjin, China PR, 2010. His major field of study is gas dynamics. 